\title{
IDENTIFICAÇÃO DE RESÍDUOS DE DISPAROS DE ARMAS DE FOGO POR MEIO DA TÉCNICA DE ESPECTROMETRIA DE MASSAS DE ALTA RESOLUÇÃO COM FONTE DE PLASMA INDUTIVO
}

\author{
Edson Luis Tocaia dos Reis, Jorge Eduardo de Souza Sarkis* e Cláudio Rodrigues \\ Instituto de Pesquisas Energéticas e Nucleares, CP 11049, 05422-970 São Paulo - SP \\ Oswaldo Negrini Neto e Sônia Viebig \\ Centro de Exames, Análises e Pesquisas, Instituto de Criminalística de São Paulo, Rua Moncorvo Filho, 410, \\ 05507-060 São Paulo - SP
}

Recebido em 29/4/03; aceito em 12/11/03

\begin{abstract}
IDENTIFICATION OF GUNSHOT RESIDUES BY HIGH RESOLUTION INDUCTIVELY COUPLED PLASMA MASS SPECTROMETRY. The violence derived from crimes involving firearms represents one of the main concerns of society. For this reason modern techniques have emerged in forensic science to identify suspects at crime scenes. This work describes a methodology to identify residues present in the hands of suspect by using a high resolution inductively coupled plasma mass spectrometry and collection procedure based on ethylenediaminetetraacetic acid (EDTA) solution as a complexing agent in moistened swabs. In order to distinguish real gunshot residues from others types of residues present in the hand of suspect, ternary ratio per cent diagrams were developed for antimony $(\mathrm{Sb})$, barium $(\mathrm{Ba})$ and lead $(\mathrm{Pb})$ detected on the hands of volunteers, before and immediately after shooting tests, revealing a remarkable difference in both situations.
\end{abstract}

Keywords: forensic science; gunshot residues; inductively coupled plasma-mass spectrometry.

\section{INTRODUÇÃo}

Nos dias de hoje, uma das maiores preocupações da sociedade refere-se à disseminação da violência e do crime organizado. É consenso que um dos principais agravantes desse quadro é o uso descontrolado de armas de fogo, que induz muitas vezes a crimes banais. Dessa forma, faz-se necessária a utilização de metodologias eficientes para a identificação de suspeitos em ocorrências criminais. Atualmente, os métodos para caracterizar tais indícios envolvem diferentes áreas do conhecimento, tais como química, biologia, física, toxicologia, informática, balística, odontologia e medicina legal. Dentre essas diversas áreas, a química desempenha um papel importante na ciência forense, notadamente a química analítica e a análise físico-química. Um exemplo é a utilização de reativos químicos para a identificação de traços de resíduos de tiros, explosões, incêndios, fibras, solos, etc. Entretanto, muitas vezes estes resíduos apresentam uma complexidade na sua composição, dificultando uma análise quantitativa completa. Apesar das técnicas utilizadas serem consagradas, é necessária uma evolução permanente destas metodologias, de modo a se obter de forma mais eficiente resultados confiáveis dos materiais encontrados. Por exemplo, em casos que envolvem armas de fogo e onde é fundamental relacionar o atirador com a arma utilizada e a vítima, técnicas de balística comumente empregadas por um exame físico de comparação de micro estrias deixadas no projétil pelo cano da arma, ou marcas características produzidas pela arma nos estojos de munição, não se aplicam diretamente ao suspeito do disparo ${ }^{1,2}$.

No momento do tiro são expelidos, além do projétil, diversos resíduos sólidos (provenientes do projétil e da detonação da mistura iniciadora e da pólvora) e produtos gasosos (monóxido e dióxido de carbono, vapor d'água, óxidos de nitrogênio e outros). Parte desses resíduos sólidos permanecem dentro do cano, ao redor do tambor e

*e-mail: jesarkis@net.ipen.br da câmara de percussão da própria arma, porém o restante é projetado para fora, atingindo mãos, braços, cabelos e roupas do atirador, além de se espalharem pela cena do crime. Deste modo, por meio dos resíduos característicos deixados pelo próprio disparo, é possível estabelecer este vínculo através de análise química das partículas encontradas. Estas partículas apresentam características peculiares, quer pela sua morfologia (Figura 1), quer pela sua composição química, onde se encontram principalmente os elementos antimônio ( $\mathrm{Sb}$ ), bário $(\mathrm{Ba})$ e chumbo $(\mathrm{Pb})^{3,4}$, provenientes de explosivos TNR (trinitroresorcinato de chumbo), de sais de bário e sulfeto de antimônio oriundos da mistura iniciadora e também da composição da liga de projéteis não jaquetados $(\mathrm{Pb}+3-4 \% \mathrm{Sb})$.

Diversas técnicas têm sido aplicadas com o objetivo de se identificar estes resíduos ${ }^{5,6}$. A Polícia Técnica de São Paulo, por exemplo, onde a análise de delitos envolvendo armas de fogo representa cerca de $80 \%$ das investigações conduzidas pelos laboratórios forenses, tem em investigações rotineiras de resíduos de disparos consagrado o uso do rodizonato de sódio como reagente colorimétrico para a identificação de chumbo, além do uso do reativo de Griess (ácido parasulfanílico) para a constatação da presença de nitritos ${ }^{1,7}$. Apesar de testes práticos e simples de serem executados, um resultado negativo em um teste colorimétrico não significa que o disparo não tenha sido efetuado: os nitritos constatados pelo teste de Griess sofrem facilmente oxidação face à ação da umidade, do oxigênio do ar e da temperatura, passando a nitratos ou volatilizando-se como ácido nitroso, prejudicando a sua identificação; por outro lado, o complexo azul-violeta, resultante da reação do rodizonato de sódio com o chumbo, pode ser estabilizado pela adição de uma solução tampão, não obstante o desvanecimento comum a complexos colorimétricos irá ocorrer como conseqüência de um contato prolongado com o meio ácido 5\% de ácido clorídrico necessário à própria reação colorimétrica, que implicará na decomposição do complexo azulvioleta em compostos incolores, podendo-se perder resultados positivos. Testes colorimétricos muitas vezes não possuem sensibilidade para detectar antimônio e bário de forma confiável, principalmente 

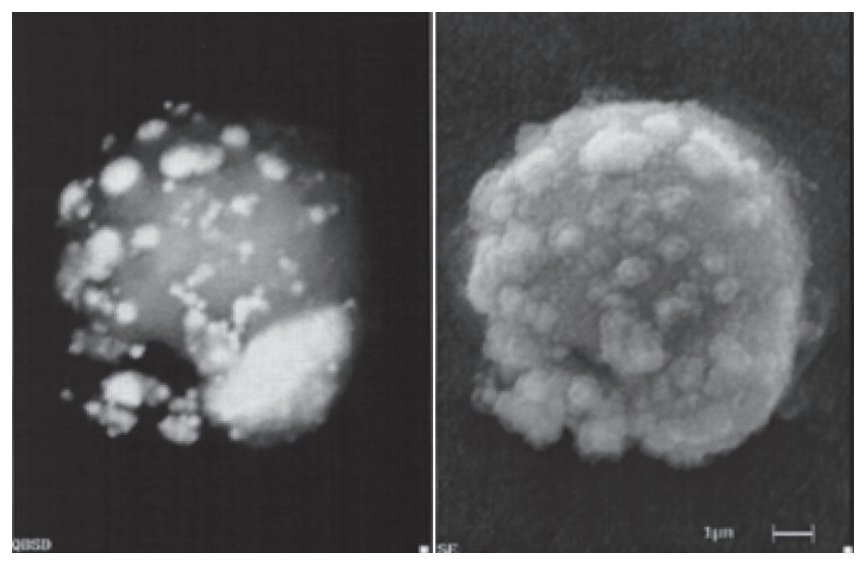

Figura 1. À esquerda, observação da morfologia de uma partícula de GSR, através de detector de elétrons secundários; à direita, imagem eletrônica obtida através do detector de elétrons retroespelhados, ambas por meio de $M E V$ - Leo 440i - com espectrômetro por energia dispersiva de raios-X com detector em estado sólido (Si/La) Oxford, com filamento de tungstênio com tensão de $20 \mathrm{Kv}$ sobre a amostra.

devido à pequena quantidade de resíduos presentes (na ordem de $\mathrm{mg}$ ) nas mãos de um atirador, bem como às suas dimensões $(0,1 \mathrm{a}$ $100 \mu$ ), o que pode limitar a detecção e identificação das partículas. Deste modo, o uso de métodos instrumentais que apresentem maior sensibilidade tem sido cada vez mais considerado. $\mathrm{O}$ uso da técnica de Microscopia Eletrônica de Varredura (MEV) acoplada à análise por raios-X por energia dispersiva, por exemplo, tem sido utilizada pelo Instituto de Criminalística de São Paulo (I.C.-SP) como técnica de confirmação de casos duvidosos ao teste do rodizonato de sódio ${ }^{1}$. Porém, em virtude do tempo requerido por cada amostra, não é utilizado em rotina.

Nos últimos anos surgiram diferentes técnicas instrumentais que têm sido cada vez mais utilizadas na área forense, aumentando a sensibilidade e a especificidade na detecção desses resíduos. A análise por ativação de nêutrons (NAA), por exemplo, remonta à década de 60, mas o fato de não detectar chumbo e a necessidade de um reator nuclear, bem como a exigência de instalações específicas, limitou o seu uso a poucos laboratórios ${ }^{2,8,9}$.

A espectrofotometria de absorção atômica (AAS) tem sido muito utilizada desde a década de 70 , com custo relativamente baixo e sensibilidade equivalente para bário e antimônio, quando comparado à técnica por NAA. Porém, essa técnica também apresenta restrições, especialmente pelo fato de ter baixo limite de detecção, além do fato de que a presença de material orgânico nas amostras pode causar dispersão de luz no feixe de radiação, em razão da sua queima incompleta durante o processo de atomização, principalmente no uso de fornos de grafite ${ }^{8,10}$. A partir de meados da década de 70 , as atenções voltaram-se para a técnica de microscopia eletrônica de varredura (SEM), capaz de analisar morfologicamente as partículas de disparos ${ }^{11,12}$. A maior limitação dessa técnica (também utilizada pelo I.C.-SP) para análises de rotina é o tempo requerido (mínimo 4 h) para se realizar cada amostra. O surgimento da técnica de espectrometria de emissão com fonte de plasma (ICP-OES) ofereceu novas vantagens relacionadas principalmente à possibilidade de análises multi-elementares; seu uso viabilizou análises de rotina, apesar de não possuir sensibilidade para a detecção de antimônio (geralmente presente em GSR) em níveis menores ${ }^{13,14}$.

Durante os últimos anos, contudo, a técnica de espectrometria de massas com fonte de plasma induzido (ICP-MS) tem se destacado como uma nova ferramenta na ciência forense. Comparativamente com as técnicas analíticas citadas anteriormente, possui maior sen- sibilidade e capacidade de proceder análises qualitativas e quantitativas multi-elementares, além de fornecer informações isotópicas, permitindo uma análise rápida e eficaz da composição inorgânica em resíduos de disparos ${ }^{15,16}$. O uso desta técnica na identificação de disparos de armas foi descrito, inicialmente, por Koons ${ }^{17}$. O método utilizado pelo autor consiste na obtenção do testemunho dos resíduos de disparo por meio de esfregaços com cotonetes na área investigada e posterior tratamento do material coletado com solução a $10 \%$ (v/v) de ácido nítrico seguido de aquecimento. O método descrito, embora tenha apresentado resultados compatíveis com outras técnicas analíticas convencionais, possui a desvantagem de recuperar apenas partículas mais expostas dos resíduos presentes nos testemunhos. Mais recentemente Reis e colaboradores ${ }^{18}$ desenvolveram uma nova metodologia para a recuperação e análise de resíduos de armas de fogo baseada no uso de cotonetes embebidos em ácido etilenodiaminotetracético (EDTA) como agente complexante, e a determinação por meio da técnica de espectrometria de massas de setor magnético com fonte de plasma indutivo.

Neste trabalho será discutido o uso desta metodologia como uma importante ferramenta analítica para identificação de assinaturas químicas características de um disparo de arma de fogo.

\section{PARTE EXPERIMENTAL}

\section{Testes de tiros}

As amostras foram obtidas por meio de voluntários que se submeteram às sessões de tiros, executadas no "túnel de tiro" do setor de Balística do I.C. - SP, utilizando armas de calibre .38 das marcas Taurus e Rossi (provenientes de apreensões da própria polícia), além de cartuchos íntegros de marca CBC (Companhia Brasileira de Cartuchos) 38SPL LRN. O uso de arma de calibre .38 e de cartuchos CBC no projeto partiu de sugestão da polícia técnica, como o tipo de arma de fogo mais apreendida, bem como o tipo de cartucho íntegro mais utilizado, respectivamente. Foram efetuados cerca de 30 disparos, sendo que cada atirador efetuou disparos com a mão esquerda e, posteriormente, com a direita. Na escolha dos voluntários, procurou-se pessoas que não tivessem tido contato anterior direto com substâncias que contivessem os elementos preponderantes $(\mathrm{Pb}$, $\mathrm{Ca}$ e $\mathrm{Sb}$ ) em resíduos de disparos, para evitar quaisquer contaminações no material a ser analisado ${ }^{19}$. As armas utilizadas foram trocadas a cada sessão de tiros e foram cuidadosamente limpas antes de cada teste de tiro, descartando qualquer possibilidade de contaminação prévia.

\section{Coleta e acondicionamento de resíduos de disparos}

A coleta foi realizada nas mãos dos atiradores por meio da técnica de esfregaço ${ }^{20,21}$, em períodos de até $1 \mathrm{~h}$ após os disparos, utilizando-se cotonetes embebidos em solução diluída de EDTA a 2\%. Aplicou-se a solução complexante ${ }^{22,23}$ em quatro regiões distintas da mão, abrangendo a palma, o dorso e a região palmar (Pinça-Palmar) e dorsal (Pinça-Dorsal) dos dedos polegar e indicador ${ }^{24}$, conforme Figuras 2(a-d).

No momento da coleta, a extremidade de um cotonete (Johnson \& Johnson) foi umedecida em uma solução de EDTA (Merck) a 2\%, durante aproximadamente $2 \mathrm{~min}$, e esfregada separadamente em cada área específica da superfície das mãos de cada atirador, por até 1 min. A extremidade do cotonete umedecida e contendo os resíduos de cada região da mão do atirador foi então seccionada e colocada dentro de um tubo de centrífuga de $15 \mathrm{~mL}$ com tampa de polipropileno (Sarstedt, Alemanha). O tubo contendo o material coletado foi devidamente identificado e levado para o laboratório. 
a)

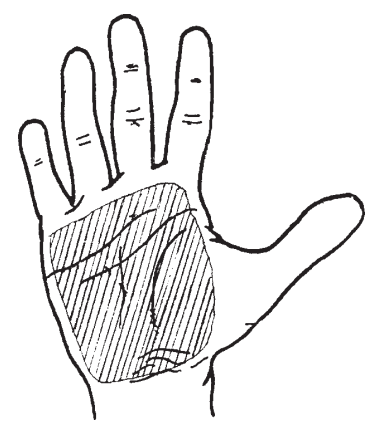

c)

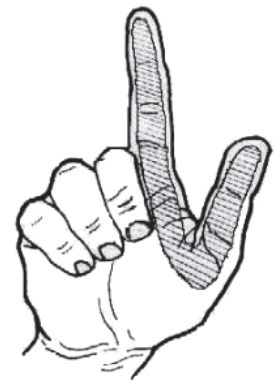

b)

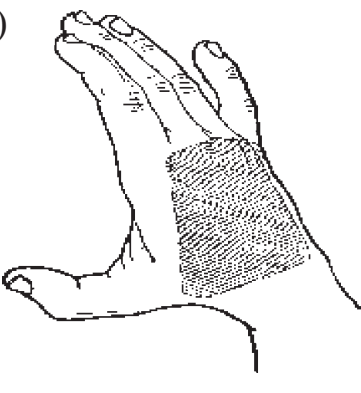

d)

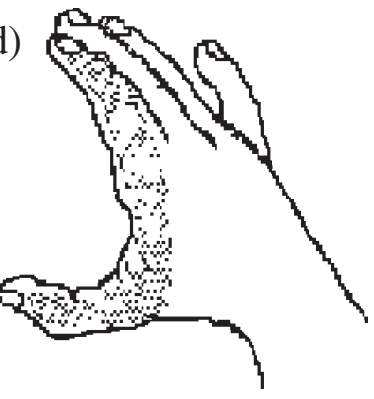

Figura 2. Regiões da mão de atiradores submetidas à coleta: a) Palma; b) Dorso; c) Região da Pinça (Palmar); d) Região da Pinça (Dorsal). Reproduzida, com permissão, da ref. 18, copyright ASTM International, 100 Barr Harbor Drive, West Conshohocken, PA19428

\section{Procedimento analítico}

Adicionou-se ao material a ser analisado um volume de $2 \mathrm{~mL}$ de uma solução a $10 \%$ (v/v) de ácido nítrico suprapuro $\left(\mathrm{HNO}_{3}\right.$; Merck) e submeteu-se durante $5 \mathrm{~min}$ a banho ultrassônico a $25 \mathrm{kHz}$ (UNIQUE, Modelo TA1800, Brasil), como forma de se favorecer a dissolução do resíduo sólido coletado. Colocou-se o tubo contendo a amostra em aquecimento de $1 \mathrm{~h}$, à temperatura de $80^{\circ} \mathrm{C}$, seguido de uma nova agitação de 5 min em banho ultrassônico. As soluções do extrato obtido foram diluídas, então, a $10 \mathrm{~mL}$ com água deionizada Milli-Q, e submetidas para análise em um espectrômetro de massas de alta energia com fonte de plasma induzido (ELEMENT 1, Finnegan MAT) para a determinação dos elementos $\mathrm{Sb}$, Ba e Pb. Um nebulizador concêntrico Meinhard em conjunto com uma bomba peristáltica foram empregados para a introdução de amostra em uma tocha de quartzo. As condições de operação principais estão relacionadas na Tabela 1 .

Tabela 1. Principais condições de operação do HRICP-MS

\begin{tabular}{ll}
\hline Fluxo gás refrigerante & $15 \mathrm{~L} \mathrm{~min}^{-1}$ \\
Fluxo gás auxiliar & $1,10 \mathrm{~L} \mathrm{~min}^{-1}$ \\
Fluxo gás amostra & $0,97 \mathrm{~L} \mathrm{~min}{ }^{-1}$ \\
Potência RF & $1300 \mathrm{w}$ \\
Cone do amostrador & Nickel, $1,0 \mathrm{~mm}$ \\
Cone do "skimmer" & Nickel $0,8 \mathrm{~mm}$ \\
Leituras por pico & 20 \\
Tempo de leitura & $0,0100 \mathrm{~s}$ \\
Janela de integração & 80 \\
Tipo de scan & Escan \\
Modo de resolução & $\mathrm{R}=300$ \\
Câmara de nebulização & Tipo Scott (PE-Sciex) \\
Tocha & Tubo de quartzo \\
\hline
\end{tabular}

\section{Padrões analíticos}

Soluções padrão multi-elementares contendo os analitos de interesse em concentrações de 1, 5, 10, 50, 100, 200 e $300 \mu \mathrm{g} \mathrm{L}^{-1} \mathrm{em}$ meio $1 \%$ de ácido nítrico foram obtidas a partir de diluições de soluções padrão originais SPEX de $\mathrm{Ba}$, Sb e $\mathrm{Pb}$, com concentração certificada de $1000 \mu \mathrm{g} / \mathrm{L}^{-1}$, por meio de uso de micropipetas (FinnpipetteLabsystems). Completou-se o volume das soluções com solução diluída de ácido nítrico suprapuro a $2 \%$. As curvas analíticas referentes a cada analito estudado foram obtidas com um coeficiente de correlação médio de $r=0,999$, e construídas em unidade de concentração $\left(\mu \mathrm{g} / \mathrm{L}^{-1}\right)$ de cada analito versus a intensidade (cps).

O limite de detecção para cada elemento em estudo, definido como a menor concentração com que um elemento pode ser detectado com $95 \%$ de confiança, e representado por três vezes o desvio padrão da intensidade das correntes iônicas obtidas na medição das soluções de branco $(\mathrm{n}=10)$, foi obtido como $0,045 \mathrm{mg} \mathrm{L}^{-1}$ para antimônio, $0,507 \mathrm{mg} \mathrm{L}^{-1}$ para bário e $0,117 \mathrm{mg} \mathrm{L}^{-1}$, para medidas em baixa resolução $(\mathrm{R}=300)$.

\section{RESULTADOS E DISCUSSÃO}

A metodologia de coleta proposta para a recuperação de resíduos de disparos mostrou ser de fácil execução, com os resultados apresentados a seguir. O procedimento analítico para a obtenção do extrato para análise também foi otimizado, de modo a simplificar ao máximo os trabalhos de rotina no laboratório.

A Tabela 2 mostra os resultados obtidos a partir de amostras coletadas das mãos de voluntários, antes e depois das sessões de tiros. As concentrações médias dos elementos $\mathrm{Sb}, \mathrm{Ba}$ e $\mathrm{Pb}$ recuperadas nas mãos dos atiradores em áreas próximas aos dedos indicadores e polegar (regiões palmar e dorsal), sugerem estas áreas específicas como as mais adequadas para se proceder a uma coleta mais provável de resíduos de disparos.

Pode-se observar também que, apesar do uso do mesmo tipo de armamento (calibre .38), a recuperação dos elementos de interesse analisados entre cada voluntário mostrou-se irregular e randômica. Quando se compara o valor médio da concentração de cada elemento com os valores máximo e mínimo obtidos nas diversas regiões amostradas da mão (Tabela 2), percebe-se que os dados analisados entre todos os voluntários não estão proporcionalmente distribuídos em torno do valor médio. A avaliação dos dados pela mediana também evidenciou esta dispersão. Esta variabilidade tem sido constatada ao longo do projeto e pode ser associada às características próprias de cada indivíduo (tamanho das mãos, atividades ocupacionais e hábitos higiênicos), assim como ao estado de conservação das armas de fogo utilizadas. A presença destes elementos, entretanto, deve ser interpretada com cautela, já que influências externas podem atuar decisivamente em uma análise final, especialmente pelo fato dos analitos estudados serem facilmente encontrados em diversas atividades ocupacionais. Estudos anteriores ${ }^{19}$ mostraram que atividades relacionadas a reparos e manutenção de automóveis, por exemplo, constituem-se em importantes fontes de contaminação de antimônio, bário e chumbo. Mesmo uma análise adicional das partículas encontradas por SEM-EDX ${ }^{21}$, de forma a se buscar uma caracterização química e morfológica do material coletado, pode também não distinguir satisfatoriamente entre partículas irregulares presentes e aquelas características de disparos, como foi mostrado na Figura 1.

A partir desta constatação, procurou-se buscar uma forma de se estabelecer uma relação entre os elementos avaliados de modo a se determinar, com maior grau de coincidência possível, a origem do material analisado como sendo exclusivamente de resíduos de disparos. Esta noção de impressão digital química, onde um conjun- 
Tabela 2. Resultados obtidos em coletas realizadas nas mãos de atiradores antes e após disparos com armas de calibre .38

\begin{tabular}{|c|c|c|c|c|c|c|c|c|c|c|c|c|}
\hline & \multicolumn{3}{|c|}{ Palma } & \multicolumn{3}{|c|}{ Dorso } & \multicolumn{3}{|c|}{ Palmar } & \multicolumn{3}{|c|}{ Dorsal } \\
\hline & $\mathrm{Pb}$ & $\mathrm{Sb}$ & $\mathrm{Ba}$ & $\mathrm{Pb}$ & $\mathrm{Sb}$ & $\mathrm{Ba}$ & $\mathrm{Pb}$ & $\mathrm{Sb}$ & $\mathrm{Ba}$ & $\mathrm{Pb}$ & $\mathrm{Sb}$ & $\mathrm{Ba}$ \\
\hline \multicolumn{13}{|c|}{ Antes-Tiro $\left(\mu \mathrm{g} \mathrm{L}^{-1}\right)$} \\
\hline Máximo & 133 & 1,13 & 98,1 & 38,7 & 1,33 & 109 & 119 & 1,30 & 146 & 52,9 & 1,27 & 140 \\
\hline Mínimo & 3,43 & $*$ & 2,90 & $*$ & $*$ & $*$ & 2,76 & $*$ & 2,22 & 2,18 & $*$ & $*$ \\
\hline Média & 29,8 & 0,35 & 24,4 & 15,2 & 0,28 & 23,1 & 38,8 & 0,35 & 25,7 & 17,3 & 0,28 & 26,0 \\
\hline Mediana & 21,7 & 0,10 & 18,9 & 12,1 & 0,06 & 18,1 & 29,8 & 0,11 & 17,9 & 13,6 & 0,04 & 16,6 \\
\hline Desvio & 30,6 & 0,39 & 21,4 & 12,8 & 0,36 & 25,7 & 31,8 & 0,41 & 29,1 & 13,7 & 0,39 & 33,5 \\
\hline \multicolumn{13}{|c|}{ Depois-Tiro $\left(\mu \mathrm{g} \mathrm{L}^{-1}\right)$} \\
\hline Máximo & 1206 & 66,6 & 236 & 731 & 81,4 & 482 & 983 & 68,5 & 298 & 7252 & 108 & 371 \\
\hline Mínimo & 12,5 & 1,25 & $*$ & 1,05 & 0,72 & $*$ & $*$ & 4,81 & $*$ & $*$ & 1,60 & $*$ \\
\hline Média & 141 & 7,71 & 33,4 & 99,7 & 9,92 & 56,3 & 257 & 19,3 & 74,8 & 475 & 22,0 & 76,8 \\
\hline Mediana & 104 & 6,64 & 11,1 & 41,4 & 3,85 & 37,7 & 162 & 13,8 & 48,6 & 123 & 15,1 & 66,9 \\
\hline Desvio & 148 & 6,83 & 55,2 & 156 & 16,7 & 93,1 & 244 & 18,4 & 89,5 & 1346 & 24,9 & 89,2 \\
\hline
\end{tabular}

* < Limite de Detecção.Reproduzida, com permissão, da ref. 18, copyright ASTM International, 100 Barr Harbor Drive, West Conshohocken, PA19428.

to de informações poderia levar a uma composição ou distribuição química redundante e definida, levou à associação dos analitos por meio do uso de gráficos ternários, que foram comparados simultaneamente em grupos de amostras coletadas antes e depois de um disparo, de forma a se constatar uma diferença nestas distribuições como um indicativo da presença de resíduos de disparos de armas de fogo em um recente evento de tiro, e estabelecer-se uma relação característica para cada situação. Os gráficos ternários constituem-se de uma comparação direta entre três componentes de um sistema. Um dado ponto, localizado em um dos vértices do triângulo, indica que a concentração do componente correspondente àquele vértice será de $100 \%$ em relação aos outros dois componentes. Por outro lado, um dado ponto localizado exatamente no centro do triângulo mostra que o percentual relativo entre os três componentes é exatamente igual.

Na Figura 3, observa-se o gráfico ternário obtido em coletas efetuadas antes dos disparos, onde cada ponto representa a relação $\mathrm{Pb}: \mathrm{Sb}$ :Ba para cada atirador. A ausência de antimônio entre os voluntários garantiu uma distribuição característica e próxima entre os atiradores. A variação de chumbo verificada poderia ser atribuída, conforme já citado, a atividades ocupacionais ou mesmo ao ambiente específico de convivência de cada voluntário.

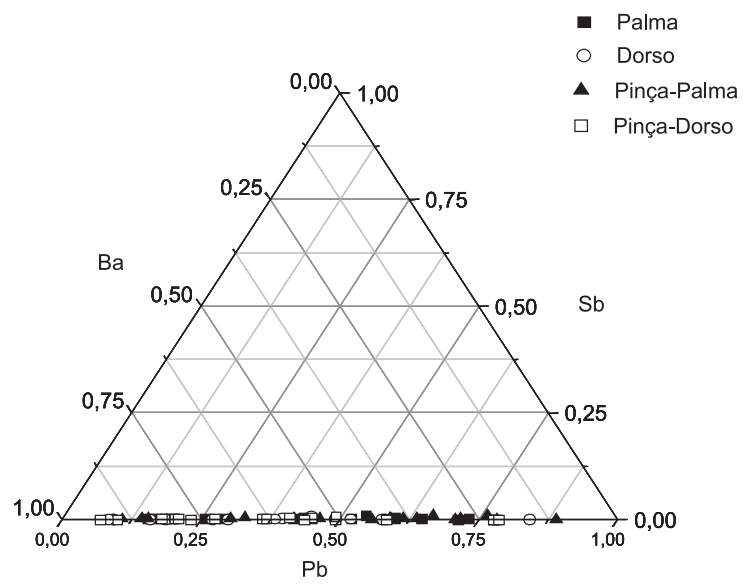

Figura 3. Relação entre $\mathrm{Sb}$, Ba e Pb encontrados nas mãos de atiradores antes dos disparos

Por outro lado, a análise dos extratos obtidos nas mãos de cada atirador depois das sessões de tiros mostrou uma diferente associação em contraponto aos analitos encontrados na Figura 3, nas mãos antes dos tiros, quando também comparados por meio de gráficos ternários, conforme pode ser visto na Figura 4.

A presença característica de antimônio, em conjunto com o aumento da concentração de chumbo, resultou em diferença significativa na relação $\mathrm{Pb}: \mathrm{Sb}$ :Ba nas duas situações estudadas, em praticamente todos os voluntários. A maioria dos voluntários apresentou, ainda, uma assinatura química similar para praticamente todas as regiões estudadas da mão, com exceção de algumas poucas razões relacionadas especialmente a coletas realizadas na região dorsal da mão de alguns atiradores. A dispersão observada nesta região específica da mão poderia, possivelmente, ser explicada pelo fato de ser uma região onde o depósito de resíduos seja menor, acarretando também menor recuperação. A comparação entre os gráficos mostrados na Figura 4 aponta, portanto, para todas as regiões da mão (com algumas ressalvas para a área dorsal) como locais mais adequados para se proceder à coleta e identificação dos resíduos, conforme o método proposto.

\section{CONCLUSÕES}

Neste trabalho, o emprego da técnica de HRICP-MS permitiu a análise em níveis de traços e ultratraços dos elementos $\mathrm{Pb}, \mathrm{Ba}$ e $\mathrm{Sb}$, a partir de um novo método proposto para coletas de resíduos de disparos de armas de calibre .38 nas mãos de atiradores, utilizandose uma solução complexante de EDTA $2 \%$.

Os resultados apresentados mostraram que as regiões ao redor dos dedos polegar e indicador constituem-se nos melhores pontos para se efetuar a coleta mais eficiente destes resíduos. Observou-se, sobretudo, que uma simples comparação das quantidades recuperadas dos analitos em alguns voluntários pode não ser decisiva para a confirmação de um tiro, especialmente quando se consideram influências externas. Porém, para a grande maioria dos atiradores, a utilização de gráficos ternários entre os analitos estudados mostrou uma relação característica no grupo de atiradores, indicando assinaturas químicas distintas nas mãos nos testes realizados antes e imediatamente depois (até $40 \mathrm{~min}$ ) de disparos com uma arma de calibre .38 .

Uma razão peculiar entre os elementos $\mathrm{Pb}: \mathrm{Sb}: \mathrm{Ba}$, constatada nas mãos de atiradores após os tiros, foi comprovada pelo aumento significativo na detecção de chumbo e, principalmente, pelo aparecimento de antimônio (aproximadamente 10 a $26 \mu \mathrm{gL}^{-1}$ ), diferenciando daque- 

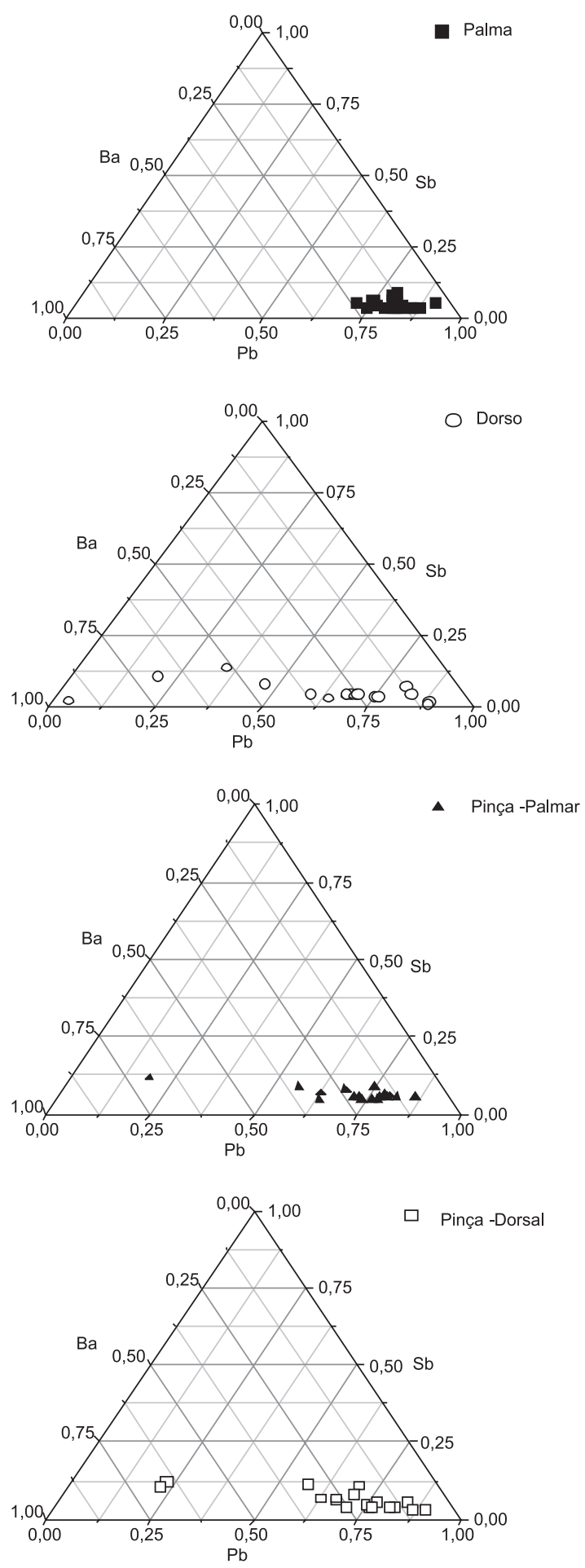

Figura 4. Gráficos ternários mostrando a distribuição entre os analitos ( $\mathrm{Sb}, \mathrm{Ba}$ e $\mathrm{Pb}$ ) em quatro regiões das mãos de atiradores (Palma, Dorso, Palma e Dorso dos Dedos Polegar e Indicador) logo após um disparo com uma arma de calibre .38 la obtida nas mãos de não atiradores (até $0,17 \mu \mathrm{gL}^{-1}$ ), e tornando-se uma poderosa ferramenta na confirmação ou não de um suspeito.

Uma análise estatística elementar baseada na média, mediana e desvio padrão não se mostrou válida para os resultados obtidos, porém a variabilidade observada nos mesmos resultados mostrou ter tido pouca importância na avaliação final dos gráficos ternários.

O método aqui apresentado para a coleta e digestão dos resíduos analisados mostrou, decisivamente, ser simples, rápido e eficaz, de forma a atender à intensa demanda da rotina da polícia de São Paulo.

\section{AGRADECIMENTOS}

Os autores agradecem aos peritos N. Correia Jr. e P. Moreira e aos funcionários do Laboratório de Balística do I.C.-SP, pelas preciosas informações durante os testes de tiros. Os autores também agradecem aos funcionários do IPEN que atuaram como voluntários nos testes de disparos, à M. H. Kakazu pelo apoio técnico e à FINEP, PADCT e CAPES pelo apoio financeiro.

\section{REFERÊNCIAS}

1. Moreira, P.; Ensaios Residuográficos e sua Aplicação no Campo da Criminalística, Núcleo de Química - CEAP - I.C.: São Paulo, 1999.

2. Ho, I.; Mat, H.; Analytical Methods in Forensic Chemistry, Ellis Horwood Limited: New York, 1990, p.40.

3. Basu, S.; J. Forensic Science 1982, 27, 72.

4. Meng, H. H.; Caddy, B.; J. Forensic Science 1997, 42, 553.

5. Singer, R. L.; Davis, D.; Houck, M. M.; J. Forensic Science 1996, 41, 195.

6. DeGaetano, D.; Siegel, J. A.; J. Forensic Science 1990, 35, 1087.

7. Bartsch, M. R.; Kobus, H. J.; Wainwright, K. P.; J. Forensic Science 1996, $41,1046$.

8. Romolo, F. S.; Margot, P.; Forensic Science International 2001, 119, 195.

9. Krishnan, S. S.; J. Forensic Science 1971, 16, 144.

10. Newton, J. T.; J. Forensic Science 1981, 26, 302.

11. Germani, M.; J. Forensic Science 1991, 36, 331.

12. Tillman, W. L.; J. Forensic Science 1987, 32, 62.

13. Koons, R. D.; Havekost, D. G.; Peters, C. A.; J. Forensic Science 1988, 33,35 .

14. Koons, R. D.; J. Forensic Science 1998, 43, 748.

15. Ulrich, A.; Moor, C.; Von Mont, H.; Jordi, H. R.; Loroy, M.; Anal. Bioanal. Chem. 2004, 378, 1059.

16. Moens, L.; Jakubowski, N.; Analytical Chemistry News \& Features 1998, 251 .

17. Koons, R. D.; J. Forensic Science 1998, 43, 748.

18. Reis, E. L. T.; Sarkis, J. E. S.; Neto, O. N.; Rodrigues, C.; Kakazu, M. H.; Viebig, S.; J. Forensic Sci. 2003, 48, 1269.

19. Garofano, L.; Capra, M.; Ferrari, F.; Brave, G. P.; Di Tullio, D.; Dell'Olio, M.; Ghitti, A.; Forensic Science International 1999, 103, 1.

20. Zeichner, A.; Levin, N.; J. Forensic Science 1995, 40, 1082

21. Zeichner, A.; Levin, N.; J. Forensic Science 1993, 38, 571.

22. Pribil, R.; Applied Complexometry, Pergamon Press: England, 1982, p. 3.

23. Welcher, F. J.; The Analytical Uses of EthyleneDiamine Tetraacetic Acid, D. van Nostrand Company: New Jersey, 1958, p.1.

24. Koons, R. D.; Havekost, D. G.; Peters, C. A.; J. Forensic Science 1987, 32,846 . 